\title{
Fracionamento das refeições e colesterol sérico em mulheres com dieta adicionada de frutas ou fibras
}

\author{
Meal frequency and serum cholesterol of women \\ in a fruit-or-fiber supplemented diet
}

Maria Conceição de OLIVEIRA'

Rosely SICHIERI²

\section{R E S U M O}

\section{Objetivo}

Estudos epidemiológicos têm mostrado uma relação inversa entre a freqüência habitual de refeições e colesterol total no sangue, sugerindo que o padrão alimentar pode modular esse lipídio. O objetivo desta pesquisa foi avaliar o efeito do fracionamento das refeições com a adição de frutas ou fibra sobre o colesterol sérico, utilizando o banco de dados de um ensaio clínico.

\section{Métodos}

Testou-se o efeito da adição de frutas ou fibras na dieta de 49 mulheres com excesso de peso (IMC $>25 \mathrm{~kg} / \mathrm{m}^{2}$ ), não fumantes, na faixa etária de 30 a 50 anos, e com colesterol sérico maior ou igual a $240 \mathrm{mg} / \mathrm{dL}$. As mulheres, alocadas randomicamente, receberam maçã $(300 \mathrm{~g})$, pêra $(300 \mathrm{~g})$, ou biscoitos de aveia $(60 \mathrm{~g})$ com igual teor de

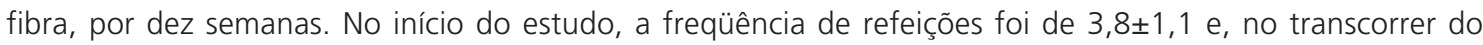
estudo, aumentou-se o número de refeições diárias para cinco. Foi então avaliada a variação no colesterol sérico segundo modificação no número de refeições diárias, calculada pela diferença entre o número de refeições no início do estudo e o número de refeições na 14a semana de intervenção. Este aumento de refeições foi categorizado em: uma, duas, três ou mais refeições diárias.

\section{Resultados}

Após 14 semanas de seguimento, observou-se que houve redução de 12,6mg/dL, $p<0,04$ e 17,4mg/dL, $p<0,03$, respectivamente, no colesterol total e no LDL-C sérico das participantes que relataram aumento

\footnotetext{
1 Departamento de Saúde Coletiva, Curso de Medicina, Faculdade de Ciências da Saúde, Universidade do Amazonas. Rua Afonso Pena, 1053, Praça 14, 69025-050, Manaus, AM, Brasil. Correspondência para/Correspondence to: M.C. OLIVEIRA E-mail: olivmc@ufam.edu.com.br

2 Instituto de Medicina Social, Universidade Estadual do Rio de Janeiro. Rio de Janeiro, RJ, Brasil.
} 
médio de três ou mais refeições diárias. Quando o modelo foi ajustado para idade, peso corporal e tipo de alimento adicionado à dieta (maçã, pêra ou aveia), as reduções foram menores $(11,5 \mathrm{mg} / \mathrm{dL}, p<0,04 ; 17,3$, $p<0,04)$; entretanto, a diferença continuou estatisticamente significante para as participantes que reportaram aumento médio de três ou mais refeições. O nível de HDL-C não mostrou alteração antes e após ajuste.

\section{Conclusão}

O fracionamento das refeições reduziu o colesterol total e LDL-C sérico em mulheres hipercolesterolêmicas, independente da idade, peso corporal e tipo de alimento ingerido, frutas ou fibras, sugerindo que o número de seis refeições diárias poderia ser uma medida de prevenção e controle da hipercolesterolemia.

Termos de indexação: colesterol, fracionamento de refeições, mulheres, dieta, estudos epidemiológicos.

\section{A B S T R A C T}

\section{Objective}

Epidemiological studies have shown an inverse relationship between people's habitual eating frequency and blood total cholesterol, leading to the suggestion that, a frequency increase in meal pattern may help to reduce the amount of such lipids. The objective was to examine data collected during a clinical trial, in order to evaluate the effects of increased meal frequency on serum cholesterol of women, who had their usual diet supplemented with either fruits or fiber.

\section{Methods}

Forty nine hypercholesterolemic women $\left(B M />25 \mathrm{~kg} / \mathrm{m}^{2}\right)$ non-smokers, ages ranging from 30 to 50 years old, and cholesterol level greater than $240 \mathrm{mg} / \mathrm{dL}$, had been tested on their usual diet supplemented with fruits or fiber, during 10 weeks. These women had been randomly assigned to add to their usual diet, either three apples (300g) or three pears (300g), or three oat-cookies (60g), all with similar fiber quantity. At baseline, the meal frequency has been 3.8.1.1; in the follow-up, the meal frequency increased to five meals per day. The registered mean of serum cholesterol was then evaluated, taking into account the change in frequency/ number of meals per day, such as one, two, three or more meals.

\section{Results}

After 14 weeks of follow-up, serum total cholesterol and $L D L-C$, each showed a reduction of $12.6 \mathrm{mg} / \mathrm{dL}$, $p<0.04$ and $17.42 \mathrm{mg} / \mathrm{dL}, p<0.03$, respectively, among participants reporting an increase of three or more meals per day. When the resulting model was adjusted for age, body weight, and type of food (fruit or fiber) added to diet, the values of total cholesterol and LDL-C changed to $11.5 \mathrm{mg} / \mathrm{dL}, p<0.04 ; 17.3, p<0.04$ respectively; however, the difference remained statistically significant for the participants that reported average increase of three or more meals. On the other hand, the HDL-C did not show any change before or after adjusting for age, body weight and type of food added to usual diet.

\section{Conclusion}

The increased number of meals reduced serum cholesterol, independently of age, body weight, and type of added food (fruits or fiber), suggesting that six meals per day may prevent and/or control hipercholesterolemia.

Index terms: cholesterol, meal frequency, women, diet, epidemiologic studies. 


\section{N T R O D U Ç Ã O}

Entre os fatores que modificam a concentração plasmática de colesterol, em homens e animais, figura a freqüência de refeições diárias ${ }^{1-3}$. Estudos epidemiológicos demonstraram que, o fracionamento das refeições diárias, em porções comidas com maior freqüência, está associado a níveis mais baixos de colesterol sérico ${ }^{4-6}$.

Mesmo em homens e mulheres com colesterol normal, o aumento do número de refeições foi capaz de reduzir este lipídio ${ }^{3,7,8}$. No estudo de Jenkins et al. ${ }^{3}$, esse efeito foi observado ao aumentarem para 17 o número de refeições diárias. Entretanto, Edelstein et al. ${ }^{4}$, ao analisarem os dados de um estudo longitudinal, observaram o mesmo efeito em indivíduos com quatro ou mais refeições diárias: observação mais compatível com as recomendações a serem incluídas em guias alimentares. O efeito do número de refeições foi também avaliado em estudos transversais ${ }^{9}$ e em ensaios clínicos controlados, tanto em homens ${ }^{10}$, como em mulheres severamente obesas ${ }^{11}$.

O consumo de fibras também pode ser importante no perfil plasmático dos lipídios, mostrando ser eficaz quando associado a uma dieta baixa em gordura e alta em carboidratos ${ }^{12}$. No entanto, o efeito das frutas sobre o colesterol total ainda não está claro. Em um ensaio clínico randômico, o consumo de frutas não alterou o colesterol total sérico ${ }^{13}$; entretanto, as frutas mostraram-se eficazes em reduzir o peso corporal $^{14}$.

O objetivo deste estudo foi testar a hipótese de que o fracionamento das refeições e aumento de sua freqüência com a inclusão de lanches de frutas ou biscoito de aveia, altera favoravelmente o nível de colesterol total sérico de mulheres com excesso de peso e hipercolesterolemia.

\section{CASUÍSTICA E MÉTODOS}

Este trabalho resulta da análise secundária de um ensaio clínico. Nesse ensaio, conduzido em mulheres sob dieta usual adicionada de frutas e biscoito de aveia, aumentou-se o número de refeições diárias, durante o seguimento de quatorze semanas do estudo, controlando-se os dados para detectar-se possíveis variáveis de confusão.

Quatrocentos e onze mulheres hipercolesterolêmicas e com excesso de peso $\left(\mathrm{IMC}>25 \mathrm{~kg} / \mathrm{m}^{2}\right)$, não fumantes, na faixa etária de 30 a 50 anos, recrutadas da Policlínica Piquet Carneiro, Universidade Estadual do Rio de Janeiro; foram convidadas a participarem do ensaio clínico randômico. Dentre elas, 51 apresentaram colesterol total sérico maior ou igual a $240 \mathrm{mg} / \mathrm{dL}$ e, destas, 49 concordaram em serem alocadas, randomicamente, para um dos três grupos: maçã (300g), com 16 participantes; pêra (300g), 16 participantes; biscoito de aveia (60g), 17 participantes. Todas foram instruídas a comerem as frutas ou biscoitos, três vezes por dia, em porções aproximadas de $100 \mathrm{~g}$ de frutas ou $20 \mathrm{~g}$ de biscoitos de aveia.

O número de refeições no início do estudo foi baixo $(3,8 \pm 1,1)$, mas a adição de frutas ou biscoitos de aveia na dieta favoreceu o aumento do número de refeições diárias das participantes, que ficou acima do habitual. O efeito desse aumento do número de refeições, sobre o colesterol sérico, foi avaliado no presente estudo.

Avaliou-se a variação do colesterol sérico segundo modificação no número de refeições diárias, avaliada pela diferença entre o número de refeições no inicio do estudo e o número de refeições na 14aㅡ semana de intervenção. Esse aumento de refeições foi categorizado em: uma, duas, três ou mais refeições diárias (diferença entre o número de refeições no início do estudo e a 14ª semana de seguimento).

A dieta ( $55 \%$ de energia proveniente dos carboidratos, $15 \%$ de proteínas e $30 \%$ de gorduras) foi elaborada com base no recordatório de 24 horas e questionário semiquantitativo de freqüência do consumo alimentar, conforme orienta Oliveira ${ }^{13}$. Para o cálculo da ingestão energética usual das participantes no início do estudo (Tabela 1), utilizou-se restrição energética 
de 230 calorias para evitar as modificações importantes no peso corporal e conseqüente alteração do colesterol total sérico. Uma dieta para perda de peso corporal, ainda que pequena, aumentaria o interesse das participantes pelo tratamento, e seu efeito sobre o colesterol total seria mínimo, enquanto que uma grande perda de peso poderia confundir o efeito do número de refeições sobre o colesterol total sérico.
Para efetuar-se a substituição dos alimentos, foram entregues a cada participante uma lista de opções de cardápio, mais o formulário para que fizesse o registro alimentar de três dias (recordando todos os alimentos consumidos a cada dia, inclusive as frutas ou bolachas de aveia e outros alimentos). Após duas semanas de estabilização, a dieta foi recalculada com a adição de maçã, pêra ou biscoito de aveia (fibras totais:

Tabela 1. Características das participantes no início do estudo.

\begin{tabular}{|c|c|c|c|c|c|}
\hline \multirow{3}{*}{ Características } & \multicolumn{2}{|c|}{1 refeição } & 2 refeições & \multicolumn{2}{|c|}{3 ou mais } \\
\hline & \multicolumn{2}{|c|}{$(n=24)$} & $(n=11)$ & \multicolumn{2}{|c|}{$(n=14)$} \\
\hline & Média & $\pm \mathrm{DP}$ & Média \pm DP & Média & $\pm \mathrm{DP}$ \\
\hline Idade (anos) & 43,3 & $\pm 6,5$ & $46,0 \pm 3,2$ & 44,7 & $\pm 5,3$ \\
\hline Renda Per capita (Reais) & 663 & \pm 483 & $933 \pm 1046$ & 455 & \pm 393 \\
\hline IMC $\left(\mathrm{kg} / \mathrm{m}^{2}\right)$ & 31,7 & $\pm \quad 3,6$ & $32,9 \pm 4,6$ & 31,5 & $\pm \quad 4,7$ \\
\hline Peso (kg) & 79,1 & $\pm 10,8$ & $80,6 \pm 11,6$ & 76,5 & $\pm 9,6$ \\
\hline Glicose (mg/dL) & 102,3 & $\pm 37,2$ & $97,5 \pm 21,9$ & 103,6 & $\pm 50,3$ \\
\hline Colesterol total (mg/dL) & 270,4 & $\pm 26,8$ & $262,1 \pm 19,7$ & 277,2 & $\pm 24,6$ \\
\hline $\mathrm{HDL}-\mathrm{C}(\mathrm{mg} / \mathrm{dL})$ & 47,4 & $\pm \quad 8,9$ & $47,7 \pm 10,9$ & 46,1 & $\pm \quad 5,8$ \\
\hline Triglicerídeos (mg/dL) & 176,8 & $\pm 69,3$ & $144,6 \pm 52,6$ & 156,8 & $\pm 74,7$ \\
\hline Número de refeições & $4,6^{\mathrm{a}}$ & $\pm \quad 0,76$ & $3,6^{b} \pm 0,67$ & $2,5^{c}$ & $\pm \quad 0,64$ \\
\hline Ingestão energética (kcal) & $3622^{a}$ & \pm 1460 & $3652^{a} \pm 1780$ & $2211^{b}$ & \pm 819 \\
\hline Ingestão de Fruta & 1,41 & $\pm \quad 0,5$ & $1,35 \pm 0,4$ & 1,18 & $\pm 0,5$ \\
\hline
\end{tabular}

Analise de variância somente para valores com diferentes letras: $a, b, c, p<0.05$.

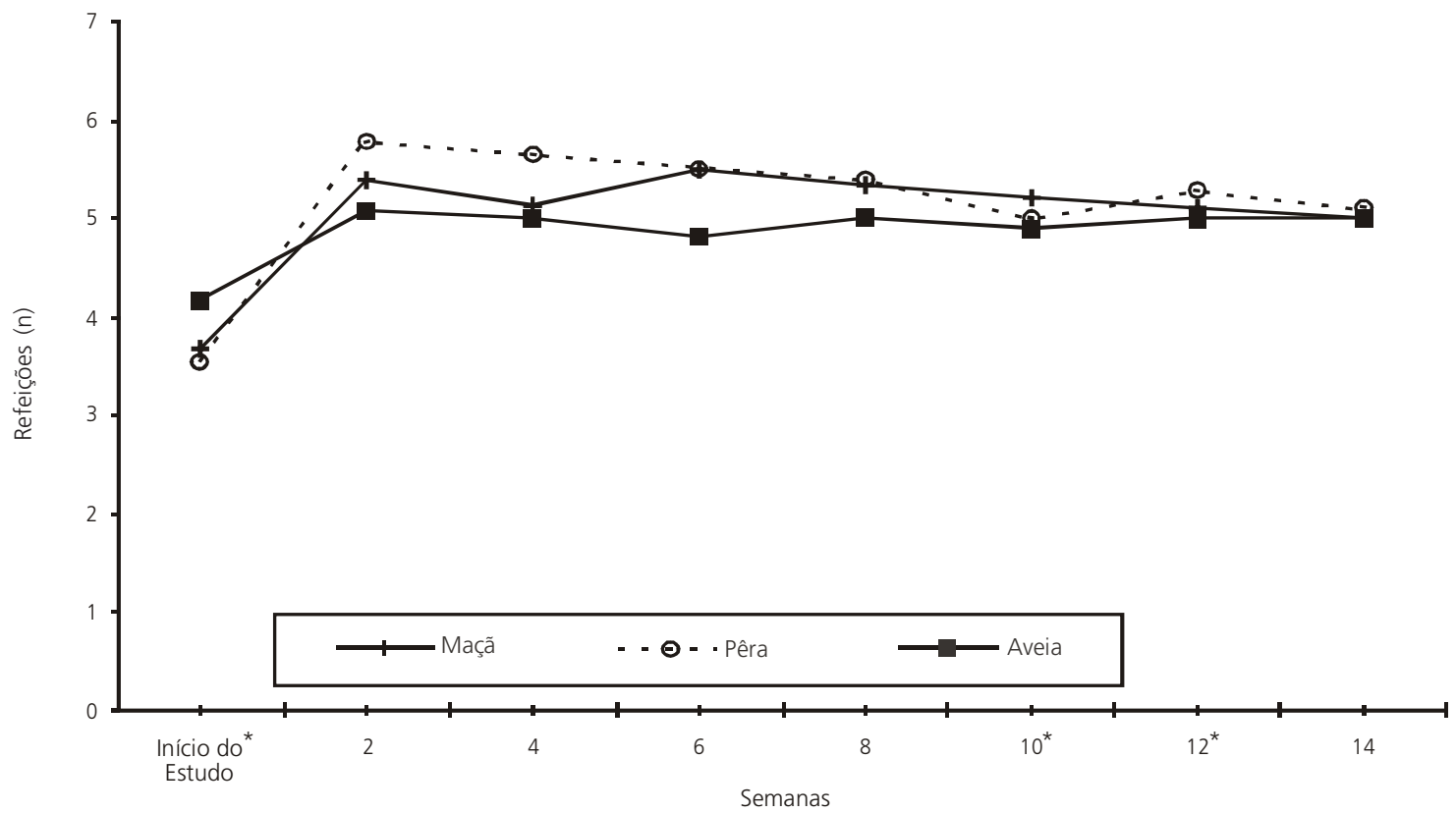

Figura 1. Freqüência das refeições nos períodos de adição e retirada de frutas e biscoitos à dieta $(p<0,05)$.

(*) Período neutro. 
6,42g, 7,32g, 6,46g; fibra solúvel: 3,0g, 1,74g, $4,09 \mathrm{~g}$ e fibra insolúvel: $3,42 \mathrm{~g}, 5,58 \mathrm{~g}, 2,51 \mathrm{~g}$, respectivamente) conforme Mendez et al. ${ }^{15}$, sendo mantida a energia prescrita na fase anterior, através da redução do feijão e do arroz. Manteve-se a dieta usual das participantes sem modificá-la qualitativamente; mas orientou-se as mesmas para que introduzissem as frutas ou biscoitos de aveia. Nessa dieta permaneceram dez semanas. No início do estudo, o número de refeições na população variou de uma a seis refeições, 3,8 1,1; no decorrer do estudo, o número médio de refeições diárias aumentou para cinco (Figura 1), ao introduzirem-se as frutas ou os biscoitos nos intervalos das refeições.

Após o período de tratamento, as frutas e os biscoitos de aveia deixaram de ser entregues, constituindo-se o (washout period) período neutro. Neste período, a dieta foi recalculada após a retirada de frutas e biscoitos de aveia, e compensada pelo aumento nas porções de arroz e feijão. No presente estudo, para efeito de análise, o colesterol sérico foi avaliado segundo três categorias de aumento do número de refeições: uma, duas, e três ou mais de três refeições diárias (diferença entre o número de refeições no início do estudo e o aumento desse número até a décima quarta semana de intervenção) como descrito anteriormente. Todas as participantes receberam as mesmas orientações dietéticas, exceto para o tipo de alimento adicionado à dieta, que diferiu nos três grupos.

O sangue foi coletado no início e a cada duas semanas durante o seguimento; as amostras de soro, após centrifugação, foram acondicionadas em temperatura de $-80^{\circ} \mathrm{C}$, para posteriores análises bioquímicas dos lipídios.

Para análise estatística, utilizou-se um método que permite levar em conta as várias medições utilizadas durante o seguimento do estudo original. Como essas medidas são correlacionadas, há procedimentos específicos que permitem analisá-las. Um desses procedimentos encontra-se no pacote estatístico Statistical Analysis System ${ }^{16}$ (SAS) e chama-se proc mixed.
Tal procedimento foi utilizado porque permitia avaliar medidas de repetição e incluir na análise as mulheres que tinham deixado o estudo ao longo do seguimento. A magnitude da alteração estimada neste modelo é obtida pelo coeficiente $\beta$ associado com o termo de interação entre o tempo e os grupos de mudança de número de refeições. O modelo foi ajustado por idade, peso e tipo de alimento adicionado à dieta (no início do estudo), para evitar confusão de dados ao associar-se o número de refeições ao nível de colesterol total sérico. A análise de variância foi utilizada no início do estudo, para comparar-se os grupos estratificados, segundo o número de refeições.

\section{RESULTADOS}

Para explorar a possibilidade das participantes que acrescentaram menos que três refeições por dia (durante o seguimento), serem diferentes das participantes que aumentaram três ou mais refeições (início do estudo, 3,8 $8 \pm 1,1$ refeições) avaliou-se outros fatores que pudessem estar associados à alteração do colesterol sérico. Nota-se que, das variáveis analisadas, apenas a ingestão energética e o número de refeições mostrou diferença significativa $(p<0,05)$. O consumo de frutas no início do estudo não diferiu nos grupos estratificados (Tabela 1).

Das 49 participantes, 34 completaram o estudo experimental, sendo um total de quinze, oito e onze participantes para cada grupo, respectivamente, que aumentaram a freqüência em uma, duas, e três ou mais refeições por dia, respectivamente (Tabela 2). Uma participante foi retirada da pesquisa, por apresentar elevado nível sérico de triglicerídeos $(1000 \mathrm{mg} / \mathrm{dL})$. Quatorze saíram ao longo da pesquisa por razões pessoais, embora a maioria tenha participado até a sexta semana.

Após a análise segundo o número de refeições, observou-se que a maioria das participantes, 49,0\% (24), aumentou uma refeição 
na dieta, enquanto $22,4 \%$ (11), aumentaram duas refeições e $28,6 \%$ (14) das participantes aumentaram três refeições ou mais. As médias e desvios-padrão do colesterol sérico segundo categoria de mudança do número de refeições, são apresentados na Tabela 2.

As participantes que aumentaram três ou mais refeições, obtiveram uma redução do colesterol total estatisticamente significante, quando comparadas às que aumentaram uma ou duas refeições. A análise dos dados mostrou que, após 14 semanas de seguimento, o colesterol total e LDL-C sérico reduziram-se $(12,6, p<0,04 ; 17,4$, $p<0,03)$ respectivamente, nas participantes que reportaram aumento médio de três ou mais refeições diárias (Tabela 3).

Tabela 2. Médias ajustadas por idade e desvio-padrão (DP) de colesterol sérico segundo o número de refeições, do início do estudo ao final do seguimento.

\begin{tabular}{|c|c|c|c|c|c|c|c|c|c|c|c|}
\hline \multirow{2}{*}{ Colesterol Total (mg/dL) } & \multicolumn{4}{|c|}{1 refeição } & \multicolumn{4}{|c|}{2 refeições } & \multicolumn{3}{|c|}{3 ou mais } \\
\hline & $n$ & Média & \pm & $\mathrm{DP}$ & $\mathrm{n}$ & Média & \pm & $\mathrm{DP}$ & $\mathrm{n}$ & Média & $\pm \mathrm{DP}$ \\
\hline Início do estudo & 24 & 270 & \pm & 27 & 11 & 262 & & 20 & 14 & 277 & \pm 25 \\
\hline Semana 2 & 24 & 251 & \pm & 32 & 11 & 254 & & 23 & 14 & 262 & \pm 39 \\
\hline Semana 4 & 19 & 242 & \pm & 27 & 11 & 258 & & 29 & 13 & 262 & \pm 25 \\
\hline Semana 6 & 19 & 259 & \pm & 46 & 10 & 259 & \pm & 37 & 12 & 258 & \pm 31 \\
\hline Semana 8 & 18 & 260 & \pm & 30 & 10 & 256 & \pm & 44 & 12 & 257 & \pm 36 \\
\hline Semana 10 & 16 & 262 & \pm & 22 & 9 & 265 & \pm & 40 & 11 & 255 & \pm 27 \\
\hline Semana 12 & 15 & 257 & \pm & 20 & 8 & 272 & \pm & 42 & 11 & 258 & \pm 32 \\
\hline Semana 14 & 15 & 261 & \pm & 32 & 8 & 248 & \pm & 23 & 11 & 257 & \pm 25 \\
\hline
\end{tabular}

n=número; DP=desvio-padrão.

Tabela 3. Alterações estimadas dos lipídios segundo aumento no número de refeições diárias.

\begin{tabular}{|c|c|c|c|c|c|c|c|c|c|}
\hline \multirow{3}{*}{ Diferença $n^{\circ}$ de refeições } & \multicolumn{9}{|c|}{ Modelo sem ajuste } \\
\hline & \multicolumn{3}{|c|}{ Colesterol mg/dL } & \multicolumn{3}{|c|}{$\mathrm{HDL}-\mathrm{C} \mathrm{mg} / \mathrm{dL}$} & \multicolumn{3}{|c|}{$\mathrm{LDL}-\mathrm{C} \mathrm{mg} / \mathrm{dL}$} \\
\hline & $\beta$ & EP & P-v & $\beta$ & EP & P-v & $\beta$ & EP & $P-v$ \\
\hline 3 ou mais & $-12,6$ & 5,95 & 0,04 & $+0,83$ & 2,73 & 0,76 & $-17,42$ & 5,60 & 0,003 \\
\hline 2 & $-3,4$ & 6,30 & 0,59 & $-0,150$ & 2,96 & 0,95 & $-3,36$ & 6,00 & 0,570 \\
\hline 1 & 0 & 0 & 0 & 0 & 0 & 0 & 0 & 0 & 0 \\
\hline Tempo & -5 & 2,50 & 0,04 & $+5,00$ & 0,42 & 0,20 & $-6,40$ & 1,96 & 0,002 \\
\hline Tempo*tempo & 0,61 & 0,28 & 0,03 & 0,056 & 0,04 & 0,19 & 0,76 & 0,22 & 0,010 \\
\hline \multicolumn{10}{|l|}{ Tempo*diferença refeições } \\
\hline 3 ou mais & $-2,40$ & 0,97 & 0,01 & $+0,240$ & 0,20 & 0,24 & $-2,97$ & 0,89 & 0,002 \\
\hline 2 & $-0,69$ & 1,05 & 0,51 & $+0,084$ & 0,22 & 0,71 & $-0,56$ & 0,97 & 0,560 \\
\hline 1 & 0 & 0 & 0 & 0 & 0 & 0 & 0 & 0 & 0 \\
\hline \multicolumn{10}{|c|}{ Modelo ajustado por idade, peso e tipo de alimento adicionado à dieta } \\
\hline 3 ou mais & $-11,50$ & 5,60 & 0,04 & $+1,28$ & 2,58 & 0,62 & $-17,30$ & 5,80 & 0,040 \\
\hline 2 & $+3,60$ & 6,30 & 0,60 & $+1,96$ & 2,99 & 0,51 & $-2,60$ & 6,50 & 0,690 \\
\hline 1 & 0 & 0 & 0 & 0 & 0 & 0 & 0 & 0 & 0 \\
\hline Tempo & $-5,20$ & 2,50 & 0,04 & $+0,58$ & 0,52 & 0,17 & $-6,40$ & 1,70 & 0,002 \\
\hline Tempo*tempo & 0,59 & 2,53 & 0,04 & 0,06 & 0,04 & 0,16 & 0,76 & 0,22 & 0,001 \\
\hline \multicolumn{10}{|l|}{ Tempo*diferença refeições } \\
\hline 3 ou mais & $-2,50$ & 0,97 & 0,01 & $+0,23$ & 0,20 & 0,25 & $-2,90$ & 0,90 & 0,002 \\
\hline 2 & $-0,64$ & 1,00 & 0,50 & $+0,33$ & 0,22 & 0,82 & $-0,52$ & 0,97 & 0,590 \\
\hline 1 & 0 & 0 & 0 & 0 & 0 & 0 & 0 & 0 & 0 \\
\hline
\end{tabular}

$E P=$ Erro-padrão; $P v=P$-valor. 
Para avaliar o efeito do número de refeições sobre o colesterol total sérico e LDL-C, independente do efeito da fruta ou fibra, foi necessário controlar esses possíveis efeitos pela variação de peso, idade e do tipo de alimentos (frutas ou biscoitos) adicionados às refeições durante o seguimento.

Após o ajuste no modelo para as possíveis variáveis de confusão, as participantes que relataram aumento médio de três ou mais refeições, acima do número de refeição habitual, mostraram redução de $11,5 \mathrm{mg} / \mathrm{dL}, p<0,04$ (Tabela 3). Enquanto que as que relataram o aumento de uma ou de duas refeições, não apresentaram alteração significativa. Também a diferença na concentração plasmática da LDL-C manteve-se estatisticamente significante $(17,3 \mathrm{mg} / \mathrm{dL} p<0.04)$ nas participantes com aumento de três ou mais refeições por dia.

Ademais, a análise mostrou aumento dos triglicerídeos nas participantes que aumentaram três ou mais refeições por dia $(35 \mathrm{mg} / \mathrm{dL}, p<0,05)$.
Após ajustar-se o modelo ao tipo de tratamento, os triglicerídeos atingiram valores ainda maiores nas participantes que reportaram aumento de três ou mais refeições $(39,8 \mathrm{mg} / \mathrm{dL}, p<0,02)$, mostrando um aumento significativo também nas participantes que reportaram aumento de duas refeições, $49 \mathrm{mg} / \mathrm{dL}, p<0,01$ - dados estes, não apresentados originalmente.

Outros dados não apresentados originalmente, foram os valores de HDL-C, que mantiveram-se inalterados para as três categorias de aumento, assim como os relativos aos níveis de glicemia de jejum, peso corporal e IMC, que não mostraram alteração significativa $(p>0,05)$.

A ingestão energética no início do estudo foi menor nas participantes que reportaram três ou mais refeições, $p<0,05$; entretanto, durante o seguimento, o consumo de energia foi similar nos três grupos estratificados por número de aumento de refeições diárias. O peso corporal, no início e ao longo do seguimento, não mostrou alterações significativas, $p>0,05$, (Figura 2).
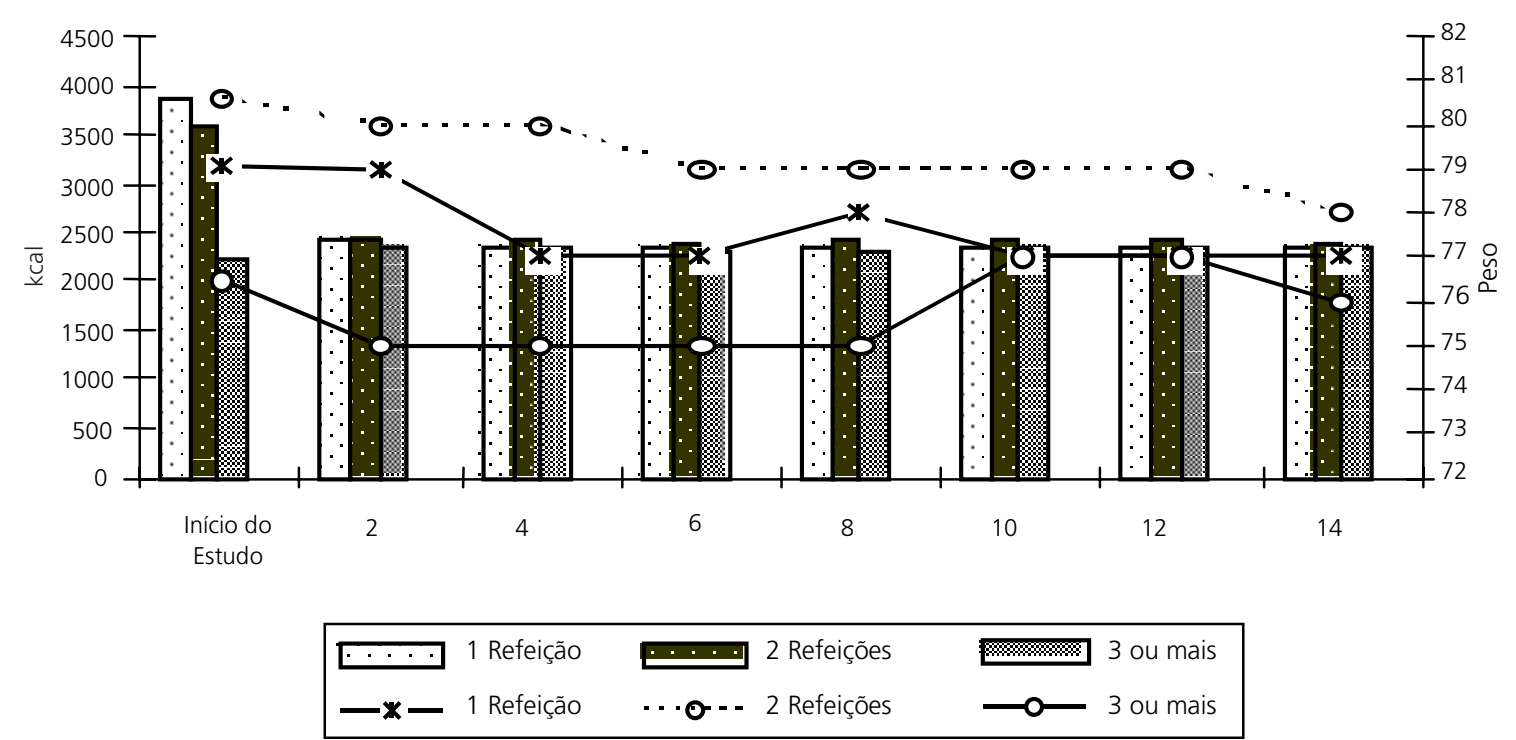

Figura 2. Ingestão energético e peso corporal, após análise dos dados segundo o número de refeições, no início do estudo e durante o seguimento ajustado para o tipo de tratamento $(p<0,05)$ e $(p>0,34)$ respectivamente.

Barras=calorias em kcal; Linhas=peso em kg.

(*) Período neutro 


\section{DISCUSSÃO E CONCLUSÃO}

A análise estratificada, segundo o número de refeições acima do habitual, mostrou redução significativa do colesterol sérico, quando o número de refeições foi acrescido, passando a três ou mais refeições por dia. Resultados similares foram observados em um estudo longitudinal, numa população de 2024 indivíduos acima de 40 anos, de ambos os sexos, analisados segundo o número de refeições: de uma a duas refeições, três refeições, quatro ou mais refeições diárias. Naqueles indivíduos com maior número de refeições as concentrações plasmáticas de colesterol e LDL-C reduziram-se, enquanto os níveis séricos de HDL-C e triglicerídeos, não foram alterados significativamente ${ }^{4}$.

No presente estudo, utilizando-se a análise segundo o aumento do número de refeições acima do habitual, observou-se redução significativa da LDL-C, para a categoria de três ou mais refeições, enquanto a análise mostrou aumento dos triglicerídeos, antes e após ajustar-se para o tipo de tratamento (frutas ou fibra). Em outro ensaio, após o ajuste, o aumento dos triglicerídeos foi também significativo, nas participantes que relataram aumento de duas refeições, evidenciando o efeito das frutas sobre os triacilgliceróis ${ }^{14}$. No presente estudo, entretanto, detectou-se também o efeito independente da freqüência/número de refeições, sobre o colesterol total, mesmo após ajuste pelas variáveis de confusão, confirmando o que foi observado por Eldelstein et al. ${ }^{4}$.

Em ensaio clínico randômico, do tipo cruzado, MacGrath \& Gibney ${ }^{8}$ compararam os efeitos de seis e os de três refeições diárias sobre os lipídios plasmáticos. Os resultados mostraram uma redução significativa do colesterol total, LDL-C e aumento da razão HDL/LDL colesterol nos indivíduos com maior número de refeições, enquanto que os níveis séricos de glicose e de triglicerídeos não se alteraram.

Na presente análise, explica-se o aumento de triglicerídeos pela adição de frutas com alto teor de frutose ${ }^{14}$. A análise da razão HDL/LDL colesterol e a glicemia não mostraram alterações significativas. O aumento da tolerância à glicose, com a ingestão de pequenas e freqüentes refeições, já foi observado em humanos ${ }^{17}$; contudo, Jenkins et al. ${ }^{3}$, utilizando modelo do tipo cruzado, com sete homens normais, mostraram redução significativa do colesterol total e LDL-C ao aumentar-se o número de refeições, sem que houvesse alteração dos níveis de glicemia e de triglicerídeos séricos.

Nem o consumo energético total, nem o consumo de fibras ou frutas parecem explicar os achados do presente trabalho, uma vez que os dados estão ajustados para a ingestão de frutas e biscoitos de aveia. No início do estudo, a ingestão energética diferiu para as três categorias de aumento do número de refeições; entretanto, durante o estudo, os três grupos apresentaram níveis de ingestão energética similares. Este fato sugere que as alterações observadas no colesterol plasmático da presente análise, não decorrem do consumo energético, da fruta ou do biscoito de aveia, e sim do fracionamento e freqüência das refeições.

Neste estudo, o consumo de frutas, comparado ao do biscoito, também não foi eficaz na redução do colesterol, como já tinha sido evidenciado por Oliveira ${ }^{13}$; quanto aos outros possíveis efeitos, estes foram controlados na análise dos dados, cujos resultados mostraram ainda pequenas alterações nos níveis de colesterol total e LDL-C séricos. A associação entre essas alterações e o aumento de três ou mais refeições diárias, mostrou-se independente do peso, da idade e do tipo de alimento adicionado à dieta, como demonstrado nos resultados que foram estatisticamente significantes, mesmo após ajustar para maçã, pêra e biscoitos de aveia, peso corporal e idade das participantes.

Outro fator que poderia estar associado à alteração do colesterol total sérico seria o consumo de frutas no início do estudo; entretanto, não houve diferença significativa no consumo entre os grupos comparados em estudos anteriores ${ }^{13}$, 
nem no consumo verificado nesta análise, após estratificação dos dados segundo número de aumento de refeições. Além do mais, os possíveis efeitos dos antioxidantes foram igualmente distribuídos, pelo menos no que se refere aos dois grupos de frutas.

As modificações no peso corporal foram semelhantes para as três categorias de aumento do número de refeições, confirmando os resultados dos estudos de Arnold et al. ${ }^{1}$ e McGrath \& Gibney ${ }^{8}$. A pequena restrição energética aconselhada na dieta (233kcal por dia) dos três grupos analisados, provavelmente resultou na pequena perda de peso observada; portanto, os dados foram ajustados segundo o peso corporal, para evitar que, mesmo essa pequena e insignificante modificação do peso corporal, alterasse os resultados.

A presente análise, considerando as quatorze semanas de seguimento e a modificação do número de refeições, mostrou redução significativa do colesterol total sérico. Novos ensaios de longa duração, com intervenção dietética e observação do efeito do fracionamento e freqüência das refeições, provavelmente confirmarão a hipótese proposta pelos resultados deste estudo.

Um possível mecanismo, através do qual o colesterol se reduziria ao aumentar-se o número de refeições, seria a alteração da síntese da enzima hidroxi metil glutaril coenzima A redutase. Esta enzima limita a produção do mevalonato, precursor do colesterol total ${ }^{1,3,7,19-21}$ e reduz a concentração plasmática de colesterol total, LDL-C e VLDL ${ }^{2,8}$.

Por outro lado, a baixa freqüência de refeições, como proposto por Fabry \& Tepperman ${ }^{21}$, favorece as alterações metabólicas que podem levar à hiperlipidemia ${ }^{7,22,23}$. O baixo número de refeições, também promove a hipertrofia do estômago e do intestino delgado, podendo contribuir para o ganho de peso ${ }^{19}$.

Nossos resultados possivelmente seriam explicados por outras características próprias das participantes, dados desconhecidos, entretanto, já que o presente estudo resulta da análise secundária dos dados registrados de um ensaio clínico. Contudo, as características que se mostraram estatisticamente diferentes entre as mulheres, no início do estudo, foram o número de refeições e o consumo energético. O menor consumo energético no início do estudo, ocorreu entre as participantes que aumentaram três ou mais refeições; entretanto esse foi o grupo que aumentou a ingestão durante o seguimento. $\mathrm{O}$ aumento do consumo de energia, por si mesmo leva ao aumento do colesterol ${ }^{24}$. Portanto, parece ser improvável que um viés de seleção esteja atuando a favor da hipótese de ausência de associação.

Em conclusão, esta análise mostrou que, mesmo uma pequena alteração para mais no número de refeições, em indivíduos com três ou menos refeições por dia, é eficaz na redução do colesterol total sérico em mulheres hipercolesterolêmicas, sugerindo que o número de seis refeições diárias poderia ser medida de prevenção e controle da hipercolesterolemia.

\section{A GRADECIMENTOS}

Os autores agradecem à Associação Brasileira de Produtores de Maçã (ABPM) pelo financiamento desta pesquisa; à Policlínica Piquet Carneiro, Instituto de Medicina Social (UERJ) e ao Hospital Universitário Pedro Ernesto, pelo suporte técnico; e também, à Coordenadoria de Aperfeiçoamento de Pessoal de Nível Superior (CAPES).

\section{REFER Ê NCIAS}

1. Arnold L, Ball M, Mann J, Mann J. Effect of isoenergetic intake of three or nine meals on plasma lipoproteins and glucose metabolism. Am J Clin Nutr 1993; 57:446-51.

2. Jenkins $D J$, Khan $A$, Jenkins $A L$, Iilingworth $R$, Pappu AS, Wolever TM, et al. Effect of nibbling versus gorging on cardiovascular risk factors: Serum uric acid and blood lipids. Am J Clin Nutr 1994; 59(3 Suppl):706-9. 
3. Jenkins DJA, Khan $A$, Jenkins $A$, Iilingworth $R$, Pappu AS, Wolever TMS, et al. Effect of nibbling versus gorging on cardiovascular risk factors: serum uric acid and blood lipids. Metabolism 1995; 44(4):549-55.

4. Edelstein SL, Barret-Cornnor EL, Wingard DL, Cohn BA. Increased meal frequency associated with decreased cholesterol concentrations; Rancho Bernardo, CA, 1984-1987. Am J Clin Nutr 1992; 55:664-9.

5. Murphy MC, Chapman C, Lovegrove JA, Isherwood SG, Morgan LM, Wrigt JW. et al. Meal frequency: Does it determine posprandial lipeamia? Eur J Clin Nutr 1996; 50:491-97.

6. Ortega RM, Redondo MR, Zamora MJ, Lopez-Sobaler AM, Quintas ME, Andres P, et al. Relationship between the number of daily meals and the energy and nutrient intake in the elderly. Effect on various cardiovascular risk factors. Nutr Hosp 1998; 13(4):186-92.

7. Jenkins DJ, Wolever TM, Vuksan V, Brighenti F, Cunnnane SC, Rao AV, et al. Nibbling versus gorging: metabolic advantages of increased meal frequency. N Engl J Med 1989; 321(14):929-34.

8. McGrath AS, Gibney MJ. The effects of altered frequency of eating on plasma lipids in free-living healthy males on normal self-selected diets. Eur J Clin Nutr 1994; 48:402-7.

9. Ma Y, Bertone ER, Stanek EJ 3rd, Reed GW, Hebert $J R$, Cohen NL, et al. Association between eating patterns and obesity in a free-living US adult population. Am J Epidemiol 2003; 158(1): 85-92.

10. Rashidi MR, Mahboob S, Sattarivand R. Effects of nibbling and gorging on lipid profiles, blood glucose and insulin levels in healthy subjects. Saudi Med J 2003; 24(9):945-8.

11. Kral JG, Buckley MC, Kissileff HR, Schaffner F. Metabolic correlates of eating behavior in severe obesity. Int J Obes Relat Metab Disord 2001; 25(2):258-64.
12. Davy BM, Davy KP, C Ho R, Besk SD, Davrath LR, Melby CL. High-fiber oat cereal compared with wheat cereal consumption favorably alters LDL-cholesterol subclass and particle numbers in middle-aged and older men. Am J Clin Nutr 2002; 76(2):351-8.

13. Oliveira MC. Modificações de peso e dos lipídios plasmáticos com dieta adicionada de frutas: ensaio clínico randomizado [tese]. Rio de Janeiro: Universidade do Estado do Rio de Janeiro; 2002.

14. Oliveira MC, Sichieri R, Moura AS. Weight loss associated with a daily intake of three apples or three pears among overweight women. Nutrition 2003; 19:253-56.

15. Mendez MHM, Derivi SCN, Rodrigues MCR, Fernades ML. Tabela de composição de alimentos. Rio de Janeiro: EDUFF; 1995.

16. Statistical Softwere, version 8.6. Cary, NC: SAS Institute; 2000.

17. Bray GA. Lipogenesis in human adipose tissue: Some effects of nibbling and gorging. I Clin Invest 1972; 51:537-47.

18. Arnold LM, Ball M, Mann J. Metabolic effects of alterations in meal frequency in hypercholesterolemic individuals. Atherosclerosis 1994; 108(2):167-74.

19. Cohn C, Joseph D. Role of rate of ingestion of diet on regulation of intermediary metabolism ("meal eating" vs. "nibbling"). Circulation 1959; 20:492-500.

20. Verboeket-Van de Venne WP, Westerterp KR. Influence of feeding frequency on nutrient utilization in man: Consequences for energy metabolism. Eur J Clin Nutr 1991; 45:161-9.

21. Jenkins DJ, Ocana A, Jenkins AL, Wolever TM, Vuksan V, Katzman L, et al. Metabolic advantages of spreading the nutrient load: Effects of increased meal frequency in non-insulin-dependent diabetes. Am J Clin Nutr 1992; 55 (2):461-7.

22. Fabry P, Tempperman J. Meal frequency: A possible factor in human pathology. Am J Clin Nutr 1970; 23(8):1059-68.

23. Gwinup G, Byron RC, Roush WH, Kruger FA, Hamwi GJ, Hamwi GJ. Effect of nibbling versus 
gorging on serum lipids in man. Am J Clin Nutr 1963; 13:209-13.

24. Goldrick RB, Hirsch J. Serial studies on the metabolism of human adipose tissue. II. Effects of caloric restriction and refeeding on lipogenesis, and the uptake and release of free fatty acids in obese and nonebese individuals. J Clin Invest 1964; 43(9):1793-804.
25. Raein-Sarjaz M, Vastone CA, Papamandjaris AA, Wykes LJ, Jones PJH. Comparison of the effect of dietary fat restriction with that of energy restriction on human lipid metabolism. Am J Clin Nutr 2001; 73(2):262-7.

Recebido para publicação em 21 de junho de 2002 e aceito em 3 de fevereiro de 2004 